\title{
CMEARTICLE
}

\section{Clinics in diagnostic imaging (153)}

Wynne $\underline{C h u a}^{1}$, MBBS, FRCR, Boon Keat $\underline{\mathrm{Lim}}^{2}$, MBBS, FRCR, Tchoyoson Choie Cheio $\underline{\mathrm{Lim}}^{3}$, FRCR, MMed
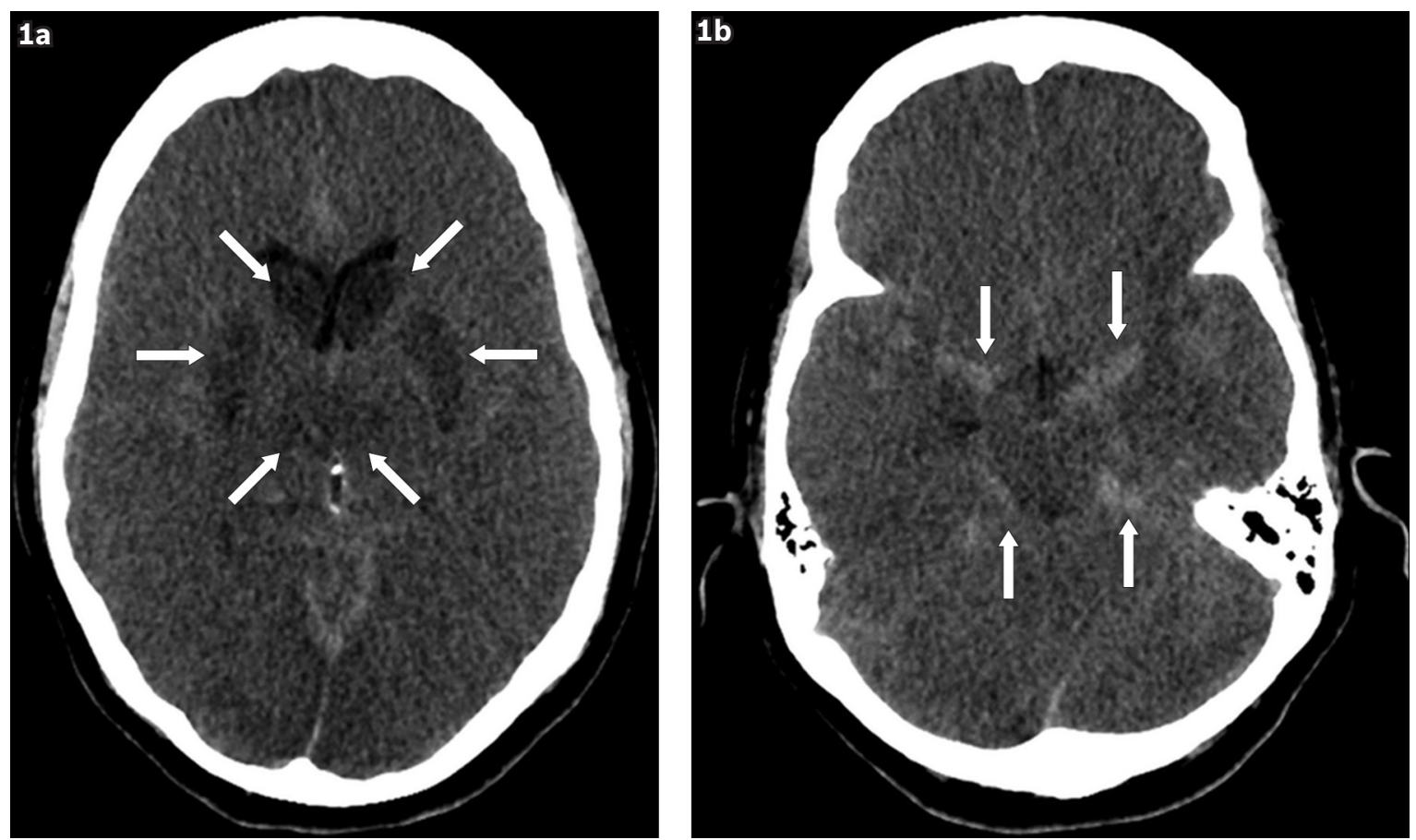

Fig. 1 Axial unenhanced CT images of the brain taken after asystolic cardiac arrest.

\section{CASE PRESENTATION}

A 58-year-old Indian woman was found lying on the floor surrounded by fresh blood, secondary to haematemesis. In the ambulance, she went into cardiac arrest, and cardiopulmonary resuscitation was started. Intravenous adrenaline was administered in the emergency department. Patient downtime was estimated to be 20 mins, after which spontaneous cardiac rhythm resumed. After resuscitation, the patient's blood pressure was at $158 / 118 \mathrm{mmHg}$, heart rate at $152 \mathrm{bpm}$ and oxygen saturation
$\left(\mathrm{SpO}_{2}\right)$ at $100 \%$. However, her pupils remained fixed and dilated, and there was no spontaneous breathing. The patient was intubated and admitted to the intensive care unit (ICU). Arterial blood gas showed severe metabolic acidosis $(\mathrm{pH} 7.03$; partial pressure of carbon dioxide [pCO $] 51 \mathrm{mmHg}$; partial pressure of oxygen $\left[\mathrm{pO}_{2}\right] 405 \mathrm{mmHg}$; bicarbonate $14 \mathrm{mmol} / \mathrm{L}$; base excess -17). Unenhanced computed tomography (CT) of the brain was done two days later (Fig. 1). What do the CT images show? What is the diagnosis?

${ }^{1}$ Department of Diagnostic Imaging, National University Health System, ${ }^{2}$ Department of Diagnostic Radiology, Khoo Teck Puat Hospital, ${ }^{3}$ Department of Neuroradiology, National Neuroscience Institute, Singapore

Correspondence: A/Prof Tchoyoson Lim CC, Senior Consultant, Department of Neuroradiology, National Neuroscience Institute, 11 Jalan Tan Tock Seng, Singapore 308433. tchoyoson_lim@nni.com.sg 


\section{IMAGE INTERPRETATION}

Axial unenhanced CT images of the brain (Figs. 1a \& b) show generalised cerebral oedema with diffuse loss of cortical grey matter-white matter differentiation, and effacement of the cortical sulci and basal cisterns. The basal ganglia and thalami are symmetrically hypodense (arrows; Fig. 1a). The sulcal spaces appear hyperdense relative to the adjacent diffusely hypodense brain parenchyma, giving rise to the pseudo-subarachnoid haemorrhage (pseudo-SAH) sign (arrows; Fig. 1b).

\section{DIAGNOSIS}

Severe hypoxic ischaemic brain injury.

\section{CLINICAL COURSE}

In the ICU, bedside oesophagogastroduodenoscopy performed on the patient revealed the presence of erosive gastritis and stale, bloodstained fluid with no active bleeding. Troponin I was recorded at 1.73 (normal range 0-0.5) $\mu \mathrm{g} / \mathrm{L}$, and two-dimensional echocardiography demonstrated a decreased ejection fraction of $25 \%$ with an akinetic left ventricular wall, which was suspicious for recent myocardial infarction. A diagnosis of severe hypoxic ischaemic brain injury secondary to cardiogenic shock was made. In the meantime, no neurological recovery was observed in the patient and her prognosis was deemed grave. The patient was terminally extubated three days later.

\section{DISCUSSION}

Severe hypoxic ischaemic brain injury in adults is often a result of cardiac arrest or cerebrovascular disease, leading to secondary hypoxaemia and diffuse brain damage. ${ }^{(1)}$ Other causes of hypoxia include asphyxiation, near-drowning and severe hypotension. . $^{(2,3)}$ Hypoxic injury to the brain, especially when severe, frequently results in profound long-term disability requiring supportive care or death from complications. ${ }^{(1)}$

The clinical features of patients with severe hypoxic ischaemic brain injury include a decreased Glasgow Coma Scale score, loss of brainstem reflexes (e.g. pupillary light, corneal, oculocephalic, gag and cough reflexes), loss of a spontaneous, normal respiratory pattern, and the presence of seizures or myoclonus status epilepticus. ${ }^{(4)}$ Electroencephalography and somatosensory evoked potential in these patients also have characteristic findings. The serum and cerebrospinal fluid levels of markers such as neuronspecific enolase, glial S-100 protein, creatine kinase and lactate may be elevated after anoxic brain injury. ${ }^{(5)}$

Hypoxic ischaemic brain injury is a systemic, metabolic derangement, and usually affects the brain diffusely, resulting in typical neuroimaging abnormalities that are bilaterally symmetrical.(3) However, these characteristic abnormalities can be subtle and difficult to discern. ${ }^{(6)}$ The typical CT features in severe hypoxic ischaemic brain injury range from subtle, generalised loss of grey-white differentiation to widespread, bilaterally symmetrical, low density generalised cerebral oedema. Grey matter, in particular the deep grey nuclei (i.e. basal ganglia, thalami), hippocampi, cerebellum and cortical grey matter

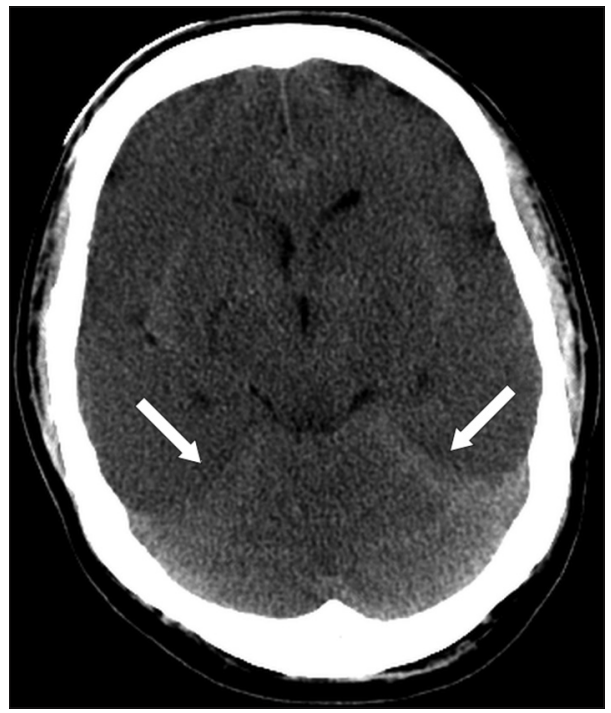

Fig. 2 A 53-year-old man who suffered cardiac arrest secondary to acute myocardial infarction. Axial unenhanced CT image of the brain shows the white cerebellum sign (arrows), denoting the appearance of a cerebellum that is hyperdense, relative to the rest of the hypodense, oedematous brain. Also, note the diffuse loss of grey-white differentiation in the rest of the brain.

(especially the perirolandic and occipital cortices) are prone to hypoxic damage and typically appear hypodense on CT.(2) Pathophysiological studies have shown the selective vulnerability of the more metabolically active grey matter compared to white matter, and the increased susceptibility of grey matter to glutamate excitotoxicity. ${ }^{(7)}$ The reversal sign is used to describe reversal of normal relative $\mathrm{CT}$ attenuation of the grey and white matter, such that the white matter is of a higher attenuation than the oedematous cortical grey matter with a lower attenuation. ${ }^{(8)}$ The pseudo-SAH sign describes relatively higher attenuation in the basal cisterns and cortical sulci on a background of diffuse, hypoattenuating brain parenchyma, mimicking the increased density of subarachnoid blood (Fig. 1b). ${ }^{(9)}$ Finally, the white cerebellum sign may be detected as diffuse, low-density oedema of the cerebrum contrasting with the relatively hyperdense normal cerebellum, caused by protective circulatory redistribution to the posterior fossa structures during anoxic events (Fig. 2). ${ }^{(10)}$

Magnetic resonance (MR) imaging may also be clinically useful in the assessment of hypoxic ischaemic brain injury, ${ }^{(11,12)}$ with conventional MR findings using fluid attenuated inversion recovery (FLAIR) and T2-weighted pulse sequences showing symmetrical hyperintensity involving the basal ganglia and cortex (detectable within three days of the event) (Fig. 3). ${ }^{(13)}$ In addition to grey matter damage, white matter hyperintensities on FLAIR or T2-weighted MR imaging representing postanoxic leucoencephalopathy may also be detected during the late subacute period (two to three weeks). ${ }^{(2)}$ In the chronic phase (more than three weeks), diffuse atrophy and cortical laminar necrosis may be detected. (2) Diffusion-weighted (DW) imaging, which has been used extensively for imaging in ischaemic stroke, depicts hyperintense changes of hypoxic ischaemic brain injury as early as one hour after occurrence. ${ }^{(2)}$ Again, these areas of restricted diffusion characteristically involve the grey matter and typically peak around 2-4.5 days ${ }^{(11)}$ and pseudonormalise by the end 


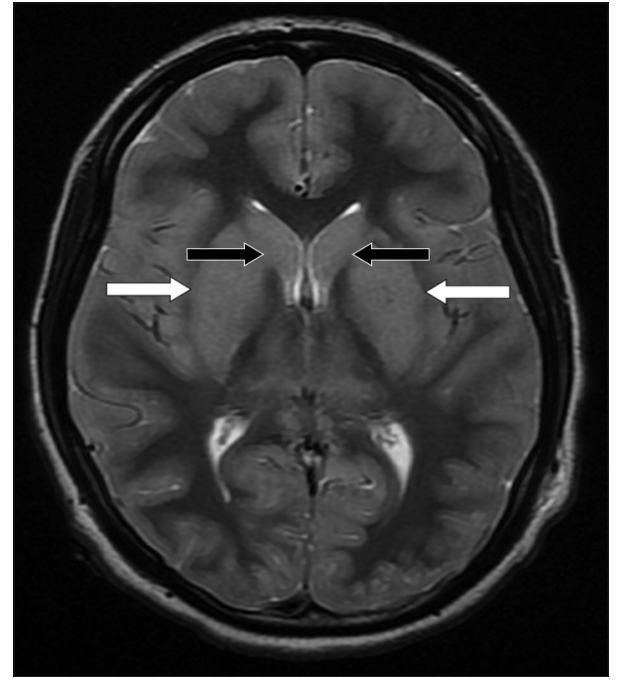

Fig. 3 A 17-year-old woman who was resuscitated after status epilepticus secondary to viral encephalitis. Axial T2-W MR image shows bilaterally symmetrical hyperintense areas in the thalamus, basal ganglia and cerebral cortex. Note the caudate nucleus (black arrows) and lentiform nucleus (white arrows) in the basal ganglia.

of the first week. Because many comatose post-cardiac arrest patients die or survive with severe disability, the cost burden to the healthcare system and family members after a protracted stay in intensive care and ensuing step-down care can be substantial. ${ }^{(14)}$ However, premature withdrawal of life support from patients who have the potential for functional recovery poses an ethical dilemma. Therefore, accurate prognostication in patients with hypoxic ischaemic brain injury becomes crucial in guiding the management of such patients. ${ }^{(11)}$ In recent studies, decreased whole-brain median apparent diffusion coefficient (ADC) ${ }^{(11)}$ and a higher percentage of brain volume with low $\mathrm{ADC}^{(12)}$ have been shown to predict poor outcome in comatose post-cardiac arrest patients, and DW imaging has been recommended for clinical prognosis in patients with hypoxia. However, MR imaging can be difficult to acquire in critically ill patients because of the need for a longer duration of image acquisition, sophisticated MR imaging-compatible monitoring equipment, and special logistical preparation within the radiological department. Recent data has suggested that CT could be an alternative to MR imaging, as the hypodensity changes on CT may be helpful for prognostication in hypoxic patients; this finding has led to some renewed interest in the use of CT techniques in such critically ill patients. ${ }^{(15)}$

In most instances, the clinical context of hypoxic ischaemic brain injury, such as out-of-hospital cardiac arrest, is readily evident and radiological diagnosis is often straightforward. However, in cases where the causative event is undocumented or ambiguous (e.g. in patients who present to the hospital in a coma of unknown cause), differential diagnosis that includes a range of systemic conditions needs to be considered. ${ }^{(16)}$ The MR imaging differentials for diffuse, bilaterally symmetrical brain damage with grey matter predilection include toxic poisoning (e.g. by carbon monoxide or methanol), systemic metabolic causes (e.g. Wilson's disease, Wernicke's encephalopathy and mitochondrial encephalopathy such as Leigh's disease), and hypoglycaemia (Fig. 4). Although the differential diagnosis for

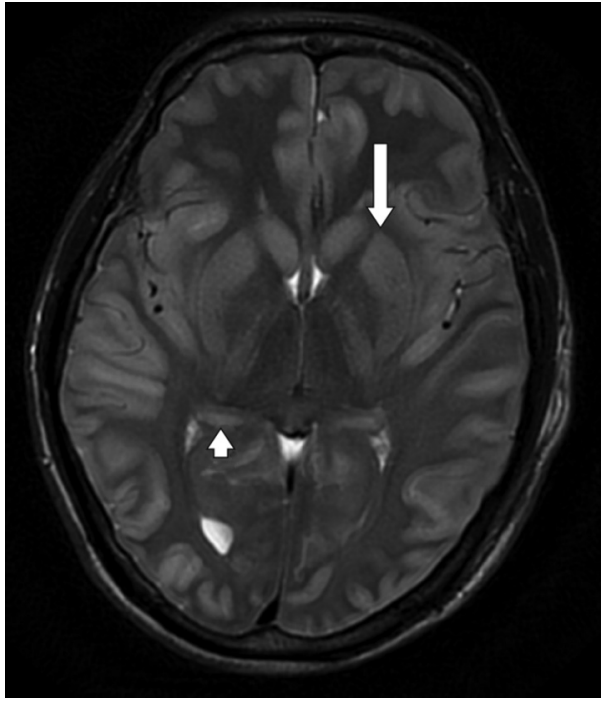

Fig. 4 A 54-year-old man who was found unconscious, with a Glasgow Coma Scale score of 4 , seizures and decerebrate posturing (from hypoglycaemia). Axial T2-weighted MR image shows bilaterally symmetrical, confluent, hyperintense lesions in the cerebral cortex, posterior hippocampus (arrowhead) and basal ganglia (arrow). The lesions spared the subcortical white matter. [Reprinted with permission from Lim et al $\left.^{(16)}\right]$.

diffuse bilateral MR imaging abnormalities can be extensive, correlation with clinical and laboratory data can help render the

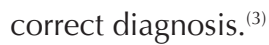

In conclusion, MR imaging and CT may be helpful in rendering the diagnosis and prognosis with regard to severe hypoxic ischaemic brain injury. Characteristic CT findings include the reversal, pseudo-SAH and white cerebellum signs, which are caused by bilaterally symmetrical decreased density, particularly of the cerebral grey matter.

ABSTRACT A 58-year-old Indian woman presented with asystole after an episode of haemetemesis, with a patient downtime of 20 mins. After initial resuscitation efforts, computed tomography of the brain, obtained to evaluate neurological injury, demonstrated evidence of severe hypoxic ischaemic brain injury. The imaging features of hypoxic ischaemic brain injury and the potential pitfalls with regard to image interpretation are herein discussed.

Keywords: cardiac arrest, hypoxic ischaemic brain injury, pseudo-subarachnoid haemorrhage, reversal sign, white cerebellum

\section{REFERENCES}

1. Huang BY, Castillo M. Hypoxic-ischemic brain injury: imaging findings from birth to adulthood. Radiographics 2008; 28:417-39.

2. Arbelaez A, Castillo M, Mukherji SK. Diffusion-weighted MR imaging of global cerebral anoxia. AJNR Am J Neuroradiol 1999; 20:999-1007.

3. Hegde AN, Mohan S, Lath N, Lim CC. Differential diagnosis for bilateral abnormalities of the basal ganglia and thalamus. Radiographics 2011; 31:5-30.

4. Levy DE, Caronna JJ, Singer BH, et al. Predicting outcome from hypoxicischemic coma. JAMA 1985; 253:1420-6.

5. Zandbergen EG, de Haan RJ, Hijdra A. Systematic review of prediction of poor outcome in anoxic-ischaemic coma with biochemical markers of brain damage. Intensive Care Med 2001; 27:1661-7.

6. Lim TCC, Nadarajah M. System-based imaging pitfalls: Brain. In: Peh WCG, eds. Pitfalls in Diagnostic Radiology. Berlin: Springer. In press. 
7. Johnston MV, Trescher WH, Ishida A, Nakajima W. Neurobiology of hypoxic-ischemic injury in the developing brain. Pediatr Res 2001; 49:735-41.

8. Bird CR, Drayer BP, Gilles FH. Pathophysiology of "reverse" edema in global cerebral ischemia. AJNR Am J Neuroradiol 1989; 10:95-8.

9. Given CA 2nd, Burdette JH, Elster AD, Williams DW 3rd. Pseudosubarachnoid hemorrhage: a potential imaging pitfall associated with diffuse cerebral edema. AJNR Am J Neuroradiol 2003; 24:254-6.

10. Harwood-Nash DC. Abuse to the pediatric central nervous system. AJNR Am J Neuroradiol 1992; 13:569-75.

11. Wijman CA, Mlynash M, Caulfield AF, et al. Prognostic value of brain diffusion-weighted imaging after cardiac arrest. Ann Neurol 2009; 65:394-402

12. Wu O, Sorensen AG, Benner T, et al. Comatose patients with cardiac arrest: predicting clinical outcome with diffusion-weighted MR imaging. Radiology 2009; 252:173-81.

13. Howard RS, Holmes PA, Siddiqui A, et al. Hypoxic-ischaemic brain injury: imaging and neurophysiology abnormalities related to outcome. QJM 2012; 105:551-61.

14. Gray WA, Capone RJ, Most AS. Unsuccessful emergency medical resuscitation--are continued efforts in the emergency department justified? N Engl J Med 1991; 325:1393-8.

15. Wu O, Batista LM, Lima FO, et al. Predicting clinical outcome in comatose cardiac arrest patients using early noncontrast computed tomography. Stroke 2011; 42:985-92.

16. Lim CC, Gan R, Chan CL, et al. Severe hypoglycemia associated with an illegal sexual enhancement product adulterated with glibenclamide: MR imaging findings. Radiology 2009; 250:193-201. 


\section{SINGAPORE MEDICAL COUNCIL CATEGORY 3B CME PROGRAMME} (Code SMJ 201407B)

Question 1. Regarding the aetiology and clinical features of patients with severe hypoxic ischaemic brain injury:

(a) Carbon monoxide poisoning is more common than cardiac arrest.

(b) In such patients, brainstem reflexes are usually preserved.

(c) Glasgow Coma Scale score tends to remain high.

(d) Death from complications and long-term disability are frequently encountered.

Question 2. In severe hypoxic ischaemic brain injury, the following are typical computed tomography (CT) findings:

(a) It may mimic subarachnoid haemorrhage.

(b) Asymmetrical hypodense basal ganglia.

(c) Diffuse effacement of cortical sulci.

(d) The white cerebellum sign is caused by cerebellar haemorrhage.

Question 3. Typical magnetic resonance (MR) imaging features of hypoxic ischaemic brain injury include:

(a) Restricted diffusion in the cortical grey matter within minutes.

(b) Hypointense diffusion-weighted imaging signal in the basal ganglia.

(c) Asymmetrical high signal in the white matter tracts on T1-weighted sequences.

(d) Sparing of the white matter at all stages.

Question 4. Regarding the prognosis of hypoxic ischaemic brain injury:

(a) Accurate prognostication can guide management decisions.

(b) CT has little value.

(c) For accurate prognostication, angiography is preferred to MR imaging.

(d) Patients with a lower whole-brain median apparent diffusion coefficient have poorer outcome.

Question 5. Regarding the typical imaging findings in hypoxic ischaemic brain injury:

(a) The imaging findings are pathognomonic and the diagnosis is confidently made based on CT or MR imaging alone.

(b) The imaging findings mimic those seen in a patient with a left-sided, chronic middle cerebral artery infarct and right-sided meningioma.

(c) The imaging findings overlap with those obtained from cases with other metabolic or toxic causes.

(d) The images should always be interpreted in the context of laboratory data and clinical history.

Doctor's particulars:

Name in full

MCR number

Specialty:

Email address

\section{SUBMISSION INSTRUCTIONS:}

(1) Log on at the SMJ website: http://www.sma.org.sg/publications/smjcurrentissue.aspx and select the appropriate set of questions. (2) Provide your name, email address and MCR number. (3) Select your answers and click "Submit".

\section{RESULTS:}

(1) Answers will be published in the SMJ September 2014 issue. (2) The MCR numbers of successful candidates will be posted online at the SMJ website by 5 September 2014. (3) Passing mark is $60 \%$. No mark will be deducted for incorrect answers. (4) The SMJ editorial office will submit the list of successful candidates to the Singapore Medical Council. 5) One CME point is awarded for successful candidates.

Deadline for submission: (July 2014 SMJ 3B CME programme): 12 noon, 29 August 2014. 